\title{
Lethal polymalformative syndrome, Boissel type
}

INSERM

\section{Source}

INSERM. (1999). Orphanet: an online rare disease and orphan drug data base. Lethal polymalformative syndrome, Boissel type. ORPHA:210144

Lethal polymalformative syndrome, Boissel type is a rare, genetic, lethal, multiple congenital anomalies/dysmorphic syndrome characterized by failure to thrive, severe developmental delay, severe postanatal microcephaly, frequent congenital cardiac defects and characteristic facial dysmorphysm (including coarse face with anteverted nostrils, thin vermillion, prominent alveolar ridge and retro- or microg natia). Additional common features include neurologic abnormalities (hyper-/hypotonia, sensorineural deafness, hydrocephalus, cerebral atrophy, seizures), as well as brachydactyly, cutis marmorata and genital anomalies. 